\title{
Addressing the Fabrication Difficulties of Femtosecond Laser Written Surface Waveguides for Enhanced Evanescent Coupling
}

\author{
Vítor A. Amorim ${ }^{1,2, *}$, João M. Maia ${ }^{1,2}$, Duarte Viveiros ${ }^{1,2}$, and P. V. S. Marques ${ }^{1,2}$ \\ ${ }^{1}$ Centre for Applied Photonics, INESC TEC, Porto 4150-179, Portugal \\ ${ }^{2}$ Department of Physics and Astronomy, University of Porto, Porto 4169-007, Portugal
}

\begin{abstract}
In this work, the fabrication of optical waveguides embedded in fused silica (Suprasil1) and boroaluminosilicate glass (Eagle2000) is demonstrated with femtosecond laser direct writing, as well as their suitability to be brought to the surface, through wet etching, for enhanced evanescent coupling with the external dielectric medium. Fused silica demonstrated to be inappropriate in this particular application, as the guiding region is at the bottom of the induced modification, creating a barrier between the guided mode and the substrate's boundary. Furthermore, the existence of nanogratings meant that, upon contact of the top of the induced modification with the substrate's boundary, the waveguide is quickly etched. Eagle2000 demonstrated to be superior to fused silica due to its characteristic modification cross-section and absence of nanogratings, which allowed the placement of the guiding region as close to the substrate's surface as required. However, surface roughness arising from the creation of insoluble products in the $\mathrm{HF}$ solution was found. The addition of $\mathrm{HCl}$ to dissolve these products was implemented.
\end{abstract}

\section{Introduction}

Femtosecond laser direct writing is a fabrication technique that relies on a very localized modification to manufacture three-dimensional structures. In several materials, the modification translates into an increased refractive index, enabling the fabrication of optical circuits [1]. The creation of nanometric structures aligned along a preferential direction (nanogratings) is also possible in fused silica, leading to an anisotropic etching reaction that enables the fabrication of microfluidic systems and, when in conjunction with optical circuits, optofluidic devices [2].

Being an inherently three-dimensional technique, it allows the fabrication of waveguides at any depth, including near the surface which is of great interest in sensing applications. However, ablation sets a limit on how shallow a waveguide can be. In this work, we employ the femtosecond laser direct writing technique in the fabrication of optical waveguides at low depths in Suprasil 1 and Eagle2000 substrates, and discuss the outcome of etching them to the substrate's surface.

\section{Experimental Procedure}

A fiber amplified femtosecond laser, with a second harmonic beam at $515 \mathrm{~nm}$ and a pulse duration of approximately $250 \mathrm{fs}$, was used to induce the refractive index modifications. These were formed by transverse scanning, with the linearly polarized beam focused at a depth of $50 \mu \mathrm{m}$ via a 0.55 numerical aperture aspherical lens. Laser beam polarization was set parallel to the writing direction, and all waveguides were written on the same scanning direction to avoid the Quill effect.

Wet etching was made in an ultrasonic bath, with the side facets of the glass substrates being polished after the etch. To determine the spectral characteristics of the waveguides, light was coupled into a single-mode fiber (SMF-28) and butt-coupled to the entrance facet of the modification tracks. The light collected by a second buttcoupled optical fiber at the exit facet was inspected in an optical spectrum analyzer.

\section{Experimental Results and Discussion}

For this study, an array of optical waveguides was fabricated in fused silica (Suprasil 1) while increasing the inscription depth in steps of $0.5 \mu \mathrm{m}$. As can be seen in Fig. 1 (a), the induced modifications' cross-section has an upper region composed by a decreased refractive index followed by a lower guiding region. To expose the guided mode to the external dielectric medium, the uppermost region must be etched. However, the presence of nanogratings translate into the etch of the laser induced modification as soon as the top of the modified region contacts the substrate's surface.

An alternative is to replace the fused silica with boroaluminosilicate glass (Eagle2000). In addition to the nonexistence of nanogratings in the laser induced modification in Eagle2000, the upper region composed by a decreased refractive index is also absent, as can be seen in Fig. 1 (b). This means that the guiding region, and consequently the guided mode, can be brought as close to the substrate's surface as desired, enabling the capability of tuning the evanescent field coupling to the exterior.

\footnotetext{
* Corresponding author: vitor.a.amorim@inesctec.pt
} 


\section{(a)}

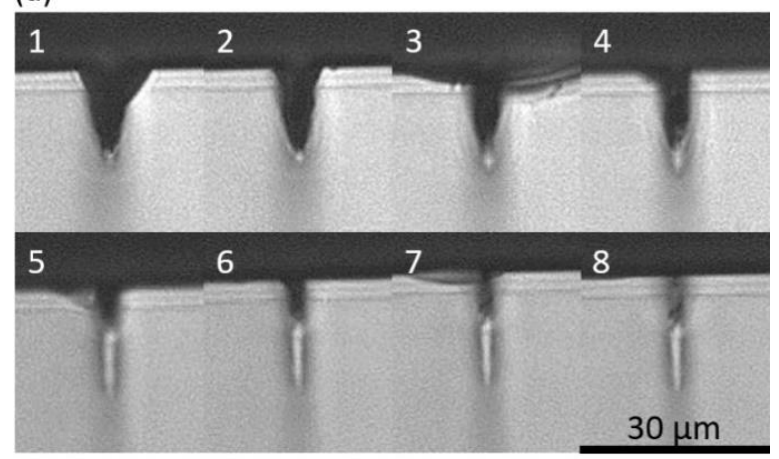

(b)

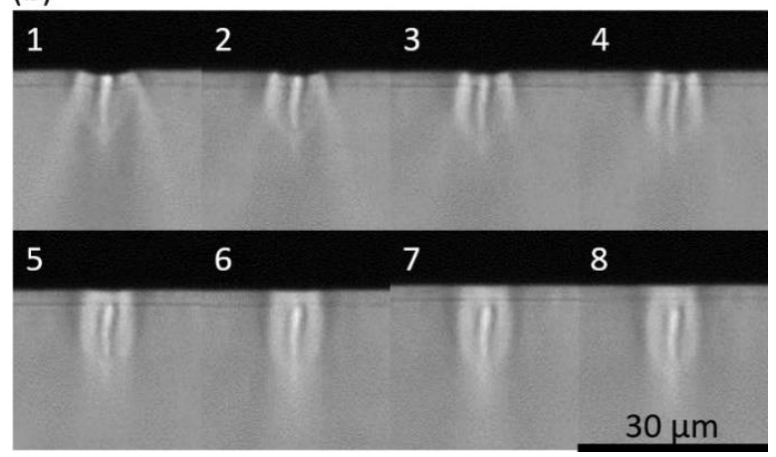

Fig. 1. Cross-section of waveguides fabricated in Suprasil 1 (a) and Eagle 2000 (b) at a depth of $50+0.5 \times n \mu \mathrm{m}$ ( $\mathrm{n}=$ waveguide number) after being etched to the surface in HF $10 \%$.

Another advantage of Eagle2000 is the lower Rayleigh scattering induced in femtosecond laser written optical waveguides when compared with fused silica [3], enabling low-loss broadband light guidance, as can be seen in Fig. 2. Their transmission characteristics also depend on the amount of etched waveguide, displaying high insertion losses at long wavelengths due to the reduced core dimensions and higher insertion losses at short wavelengths due to reduced coupling efficiency and increased Rayleigh scattering from nanometric defects at the substrate's surface.

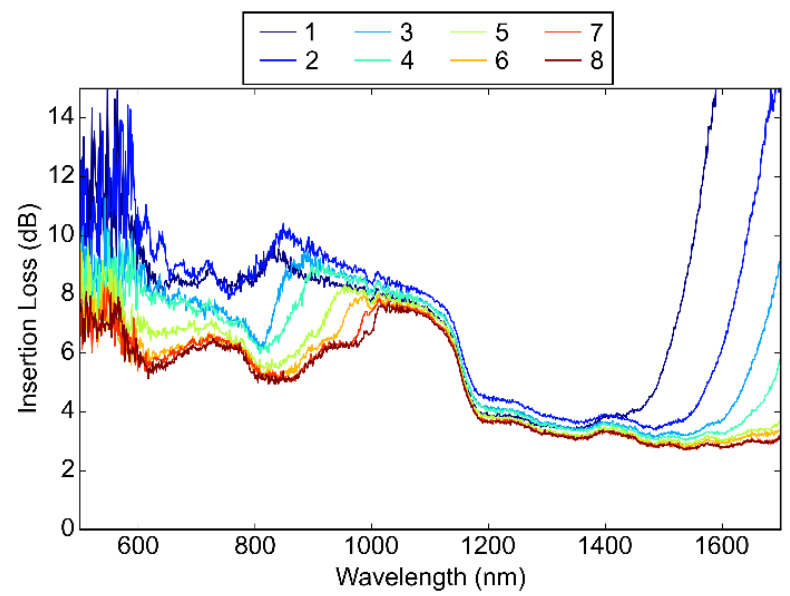

Fig. 2. Insertion loss of $2.4 \mathrm{~cm}$ long waveguides fabricated in Eagle 2000 at a depth of $50+0.5 \times n \mu \mathrm{m}$ (where $\mathrm{n}$ is the waveguide number) after being etched to the surface in $\mathrm{HF} 10 \%$.

Fig. 3 displays the surface topography of Eagle2000 before and after etching with HF $10 \%$. As can be seen, the surface roughness increases significantly after etching, limiting possible applications such as the deposition of metallic thin-films for plasmonic sensors. The roughness is connected with the creation of insoluble products in the HF solution, which deposit on the generated surface and act as a masking layer [4]. By adding $\mathrm{HCl}$ the products become soluble, improving the etched surface's quality, as visible in Fig. 3 for a solution of $\mathrm{HF} 1 \% / \mathrm{HCl} 37 \%$ for a volume ratio of $10 / 1$.

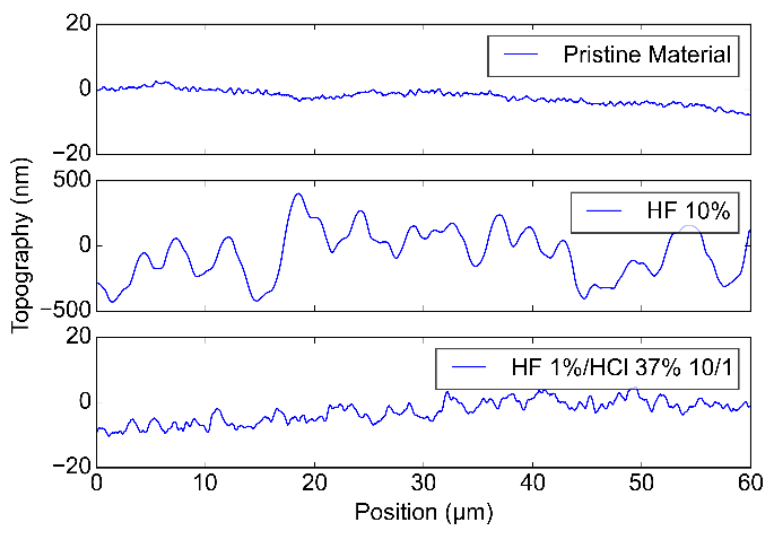

Fig. 3. Surface topography of Eagle2000 with a pristine surface, after being etched in $\mathrm{HF} 10 \%$, and after being etched in $\mathrm{HF} 1 \% / \mathrm{HCl} 37 \%$ for a volume ratio of $10 / 1$.

\section{Conclusions}

Eagle2000 has shown to be suitable for the fabrication of surface waveguides using femtosecond laser direct writing and wet etching. The nonexistence of nanogratings in the laser induced modification and the absence of an upper region composed by a decreased refractive index, as in fused silica, enables the placement of the guiding region as close to the substrate's surface as desired. Furthermore, waveguides fabricated in this glass present low-loss broadband guiding characteristics, suiting a broad range of applications. Increased surface roughness, created by insoluble products when etching with HF, was identified, being minimized with the application of $\mathrm{HCl}$ in the etchant solution.

This work was supported by Fundação para a Ciência e a Tecnologia through grant no. SFRH/BD/128795/2017 and by project "NanoSTIMA: Macro-to-Nano Human Sensing: Towards Integrated Multimodal Health Monitoring and Analytics/NORTE-01-0145-FEDER-000016", financed by the North Portugal Regional Operational Programme (NORTE 2020), under the PORTUGAL 2020 Partnership Agreement, and through the European Regional Development Fund (ERDF).

\section{References}

1. V. A. Amorim, J. M. Maia, D. Alexandre, P. V. S. Marques, J. Light. Technol. 357 (2017)

2. J. M. Maia, V. A. Amorim, D. Alexandre, P. V. S. Marques, J. Light. Technol. 358 (2017)

3. V. A. Amorim, J. M. Maia, D. Viveiros, P. V. S. Marques, J. Light. Technol. 6 (2019)

4. C. Iliescu, J. Jing, F. E. H. Tay, J. Miao, T. Sun, Surf. Coat. Tech. 1985 (2005) 